\title{
A Fibroblast-Derived Human Growth Factor Preparation for the Management of Acute Radiodermatitis: A Case Report
}

This article was published in the following Dove Press journal: International Medical Case Reports Journal

\author{
Georgia Vadarli' \\ Maria Angelo-Khattar (iD) ${ }^{2}$ \\ 'Plastic Surgery Consultancy Private \\ Practice, Thessaloniki, Greece; \\ ${ }^{2}$ American Academy of Anti-Aging \\ Medicine (A4M), Dubai, United Arab \\ Emirates
}

\begin{abstract}
Radiotherapy is a commonly used modality in breast cancer management. Amongst the consequences of treatment are acute and/or chronic skin changes, which often lead to itching, pain, and a diminished aesthetic appearance, all of which greatly impact the patient's quality of life. Management of radiation-induced skin effects has not as yet been standardized, with various protocols being applied by different radiotherapy departments. This case study outlines the management of a grade 3 acute radiodermatitis in a female with a synergistic preparation of anti-inflammatory cytokines and growth factors derived from human fibroblasts. The treatment resulted in the total resolution of the radiation-induced skin reaction, with a minimally visible residual scar.
\end{abstract}

Keywords: radiotherapy, acute radiodermatitis, growth factors, cytokines

\section{Introduction}

It is estimated that radiotherapy (RT) is administered in more than half of patients diagnosed with cancer. The goal of the treatment is to deliver optimal results with the least possible untoward effects. ${ }^{1}$ Nonetheless, it has been reported that even with the most advanced radiotherapy procedures, approximately $95 \%$ of patients will experience substantial skin reactions in the treatment area.,

These untoward effects on the skin, secondary to radiotherapy, may be acute or chronic. Acute radiodermatitis is a burn injury, which generally appears within one to four weeks after treatment initiation and persists for the radiotherapy duration. Chronic or late-onset reactions may appear in several years post-therapy. The severity of radiodermatitis has been graded by the National Cancer Institute (USA) into four levels: Grade 1 (mild erythema or desquamation), grade 2 (moderate erythema and moist desquamation confined to skin folds and creases), grade 3 (confluent moist desquamation greater than $1.5 \mathrm{~cm}$ diameter which is not confined to skin folds possibly with pitting oedema) and grade 4 (skin necrosis or ulceration of full thickness of the dermis). ${ }^{4,5}$

At present, there is no unanimous agreement regarding recommendations to prevent or treat radiodermatitis despite the publication of general guidelines by several multidisciplinary groups. ${ }^{6-8}$ In fact, substantial variations exist regarding patient instructions, specified by various radiotherapy units. ${ }^{9}$ Nonetheless, it is generally agreed that maintaining a clean and dry irradiated area and diligent moisturization of the treated area with the use of steroid creams and skin barrier preparations are beneficial in mitigating the skin response to radiation.
Correspondence: Maria Angelo-Khattar POB 5956, Dubai, United Arab Emirates $\mathrm{Tel}+97 / 506245494$

Emailmkhattar@younatagroup.com
International Medical Case Reports Journal 2020:13 69|-696 
Since the primary reasons for acute radiodermatitis skin reactions are inflammation, a reduction in the number of stem cells and skin-cell necrosis and death, ${ }^{10}$ it stands to reason that treatment of radiodermatitis wounds with antiinflammatory cytokines and growth factors may be beneficial in modulating the inflammatory response and regulating an orderly wound-healing reaction. It is a wellestablished fact that growth factors and cytokines are integral to each of the three phases of normal wound healing, namely inflammation, proliferation, and matrix remodeling. Levels of growth factors, such as PDGF, EFG, TGF-Beta and FGF, are reduced in chronic pressure and dermal ulcers than acute wounds. ${ }^{11,12}$ Each of these growth factors has a specific role in the promotion of wound healing. TGF-Beta stimulates collagen and glycosaminoglycan formation, increases fibronectin synthesis, inhibits matrix degradation and facilitated cell chemotaxis. Platelet-derived growth factor stimulates angiogenesis and is a potent activator for cells of mesenchymal origin. Epidermal growth factor is responsible for the regeneration of the epidermis and fibroblast growth factor stimulates fibroblasts and angiogenesis.

Exogenous application of a synergistic complex of anti-inflammatory cytokines and growth factors may be of potential benefit in the regenerative process leading to orderly wound healing of radiodermatitis.

\section{Patients and Methods}

\section{Case presentation}

A 49-year-old female nurse was treated with radiotherapy for HER2 breast cancer. The patient was otherwise healthy with no co-morbidities. The patient developed an area of thin weeping skin, greater than $1.5 \mathrm{~cm}$ in diameter, which was not confined to skin folds (Grade 3 radiodermatitis) in the right clavicle area.

Written informed consent has been provided by the patient to have the case details and any accompanying images published.

\section{Case History}

The patient had undergone a right-sided partial mastectomy on 30 July 2019 and began radiotherapy two weeks later. She underwent a total of twenty-five RT sessions at a frequency of five per week.

Upon initiating the RT sessions, the patient was advised to use a regenerative cream containing $10 \%$ zinc oxide, D-panthenol and allantoin (Zinco cream), by her treating physician. The patient was instructed to apply the cream twice a day, as of the first RT session. No further recommendations were given by the radiotherapy department. Despite the diligent use of the Zinco cream, the patient presented with mild redness across the region of her right breast, which extended to the ipsilateral clavicle area. Whilst the erythema remained unchanged on the irradiated breast, the region of the right clavicle presented with an acute radiation burn during the 22nd RT session (Figure 1).

\section{Physical Examination}

Upon developing the skin reaction, the patient was referred to a Plastic Surgeon, Dr. Vadarli (author), for consultation. However, as the patient lived in a provincial town and had no means of transportation, she opted for online consultation with the plastic surgeon.

The plastic surgeon diagnosed a confluent area of moist desquamation $(10.0 \mathrm{~cm}$ long, $3.0 \mathrm{~cm}$ wide) in the right clavicle area during the online consultation.

\section{Treatment Plan}

The patient was instructed to clean the affected area twice a day with saline solution, dry it with sterile gauze, and apply a single dosimeter-press dose of synergistic anti-inflammatory preparation cytokines, interleukins, and growth factors (Active Serum by AQ Skin Solutions, Irvine, California, USA). The patient was instructed to use the AQ active serum twice a day during the $23^{\text {rd }}, 24^{\text {th }}$ and $25^{\text {th }}$ RT sessions and for a further four days post-RT. Thereafter, she was instructed to use the serum continuously, once a day.

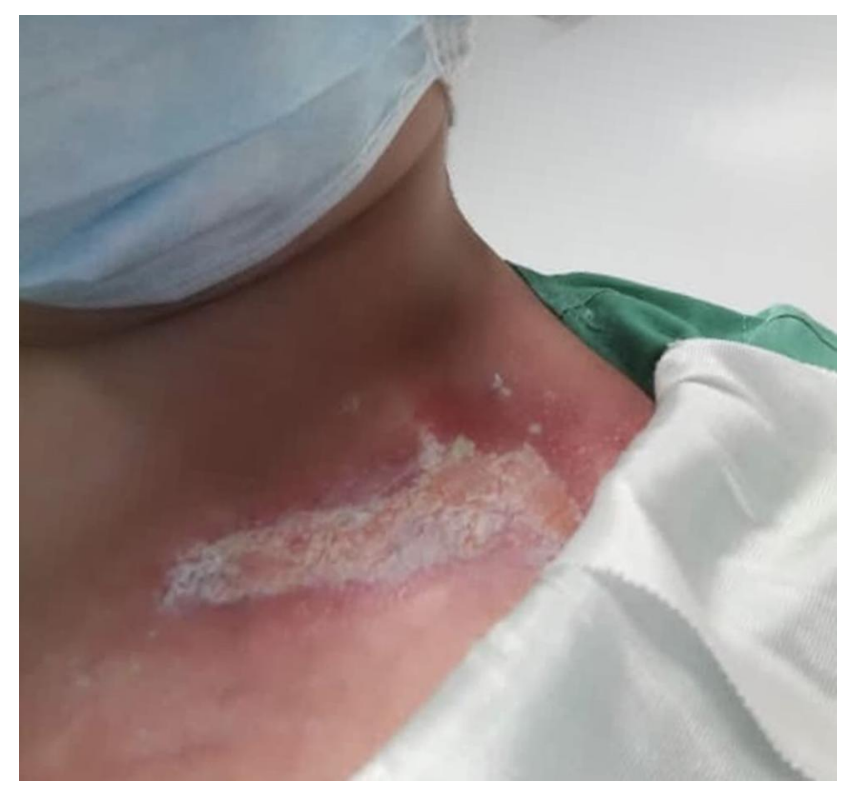

Figure I Day I post the $22^{\text {nd }}$ RT session. Day I AQ serum therapy. Pain score $=7$. ARD grade 3 . 


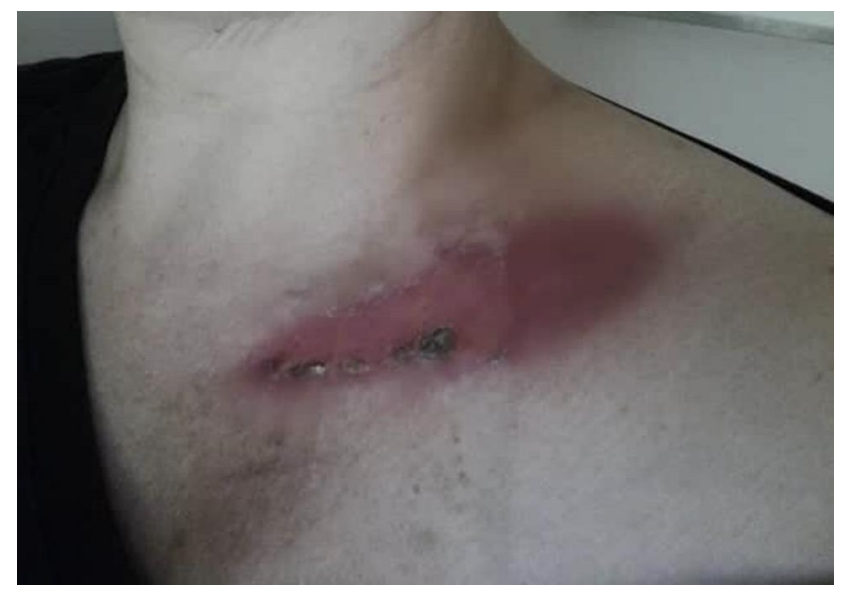

Figure 2 Day I post $23^{\text {rd }}$ RT treatment. Day 4 of AQ therapy. Pain score=3. ARD grade 2 .

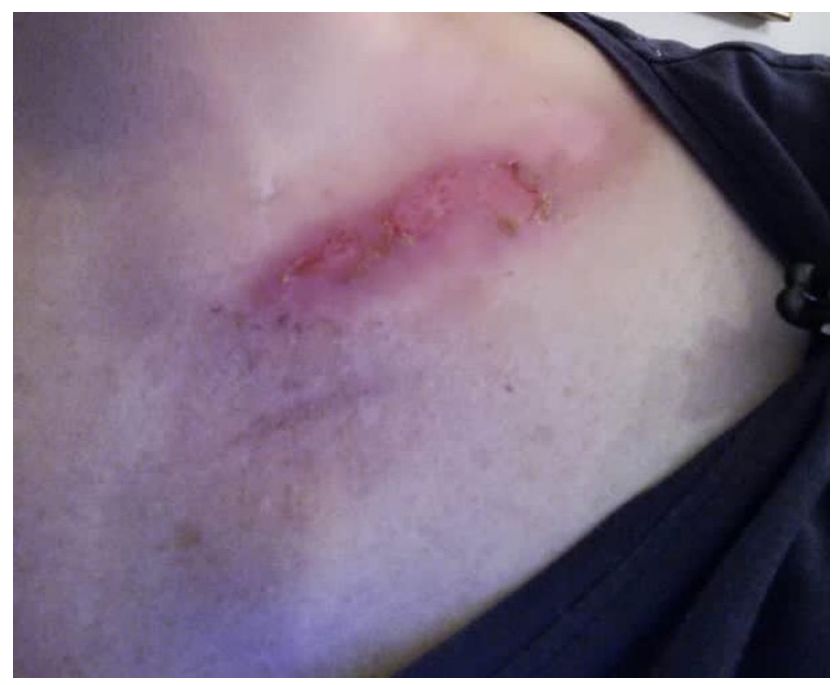

Figure 3 Day 6 post $24^{\text {th }}$ RT. Day 9 post-AQ therapy. Pain score $=2$. ARD grade 2 .

The preparation derived from cultured human fibroblasts contains Transforming Growth Factors (TGF) Beta-1, 2 and 3, Platelet-Derived Growth Factor (PDGF), Granulocyte Monocyte Colony Stimulating Factor (GMCSF), and Interleukins (IL) 3, 6,7 and 8.

On the tenth day of treatment, the outflow of serous fluid ceased, and the burn area was subsequently treated once a day.

The patient continued with three further RT sessions, although the burn wound remained open throughout the treatment sessions.

The patient's progress was monitored online, and she photographed the wound herself regularly (Figure 2-8). The patient was requested to maintain a record of her pain score on a scale of 1 to 10 .

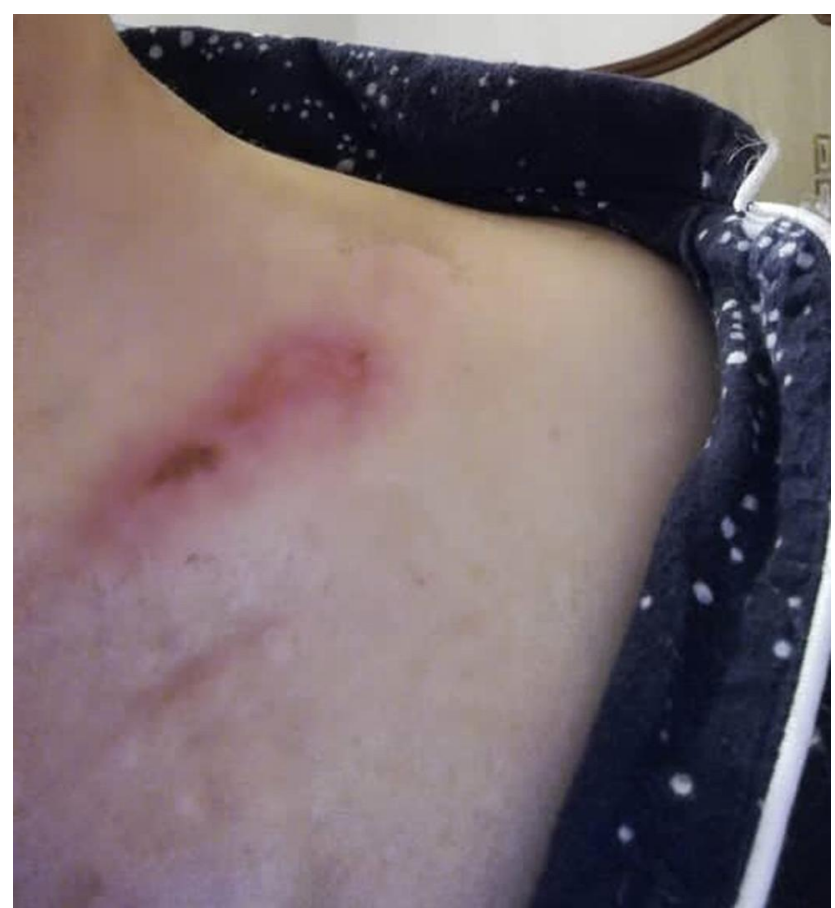

Figure 4 Day II post $25^{\text {th }}$ RT. Day I4 post-AQ therapy. Pain score=I. ARD grade 2.

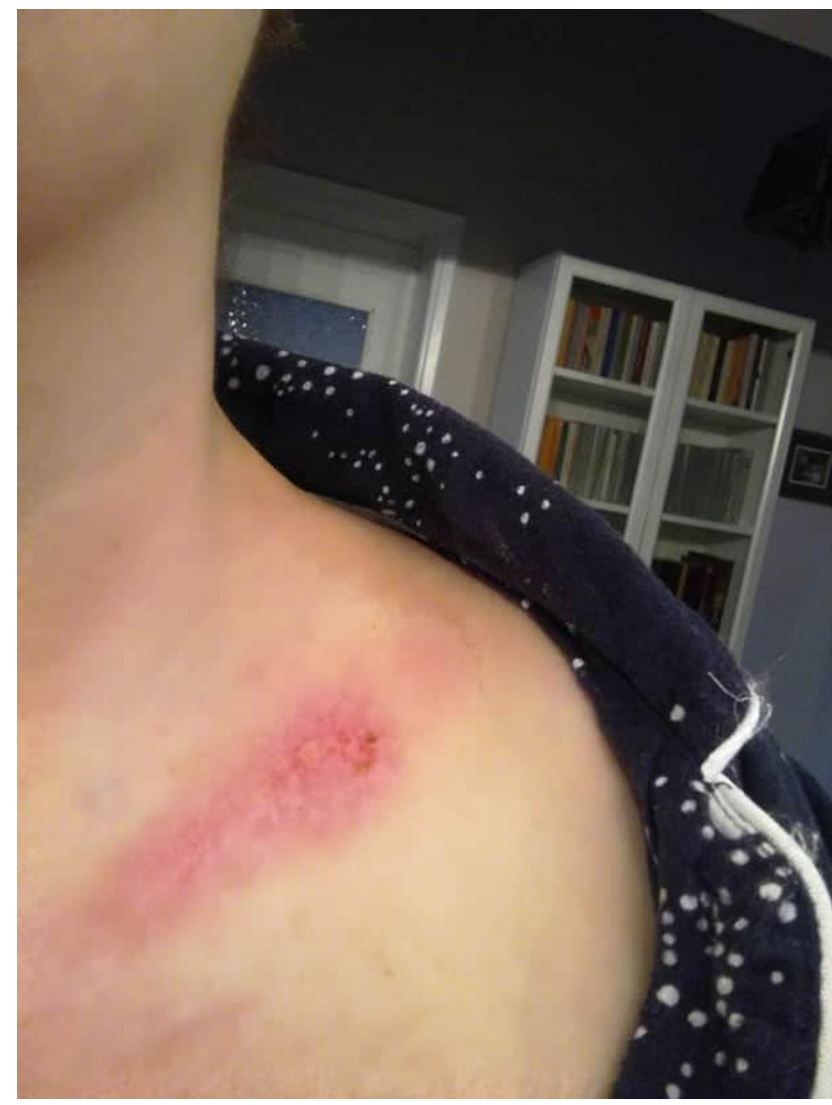

Figure 5 Day 12 post $25^{\text {th }}$ RT. Day 15 post-AQ therapy. Pain score=I. ARD grade I. 
According to the patient, the AQ Active Serum was easy to apply and acted as a protective film. Although the patient did not apply any additional dressing, the serum offered her protection against contamination. Furthermore, her clothes did not adhere to the burn area, and she experiences a significant reduction in pain and observed a clear reduction in the erythema.

The final photo six months after treatment was taken by her plastic surgeon in the clinic (Figure 9).

\section{Result of Treatment}

The treatment outcome was that the radiation-induced skin reaction responded to treatment relatively quickly, within 21 days of initial daily application of the AQ Active Serum by the patient, with no additional treatment or dressing. The grading of ARD at each time frame post-RT, the day of AQ serum application and the pain score at each stage are shown in Figures 1-9.

The patient did not experience any complications and was relatively pain-free during the recovery. The outcome

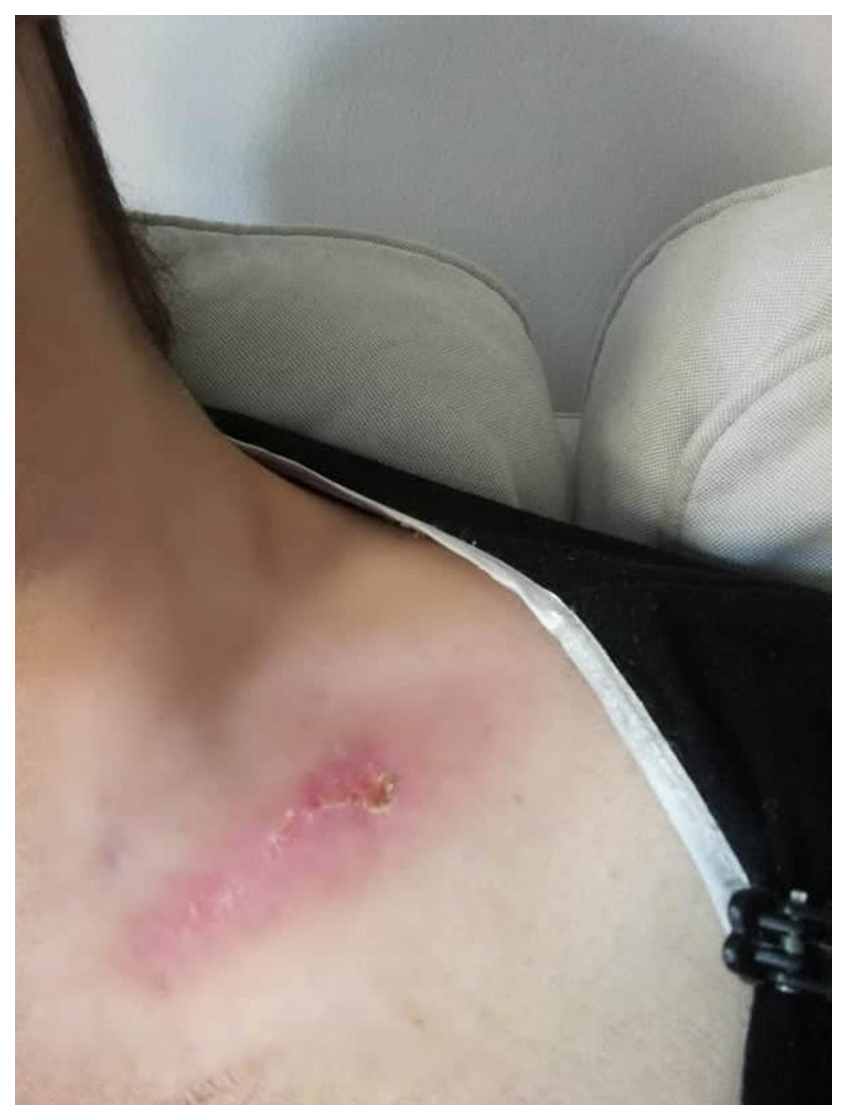

Figure 6 Day 14 post-RT. Day 17 post-AQ therapy. Pain score=I. ARD grade I.

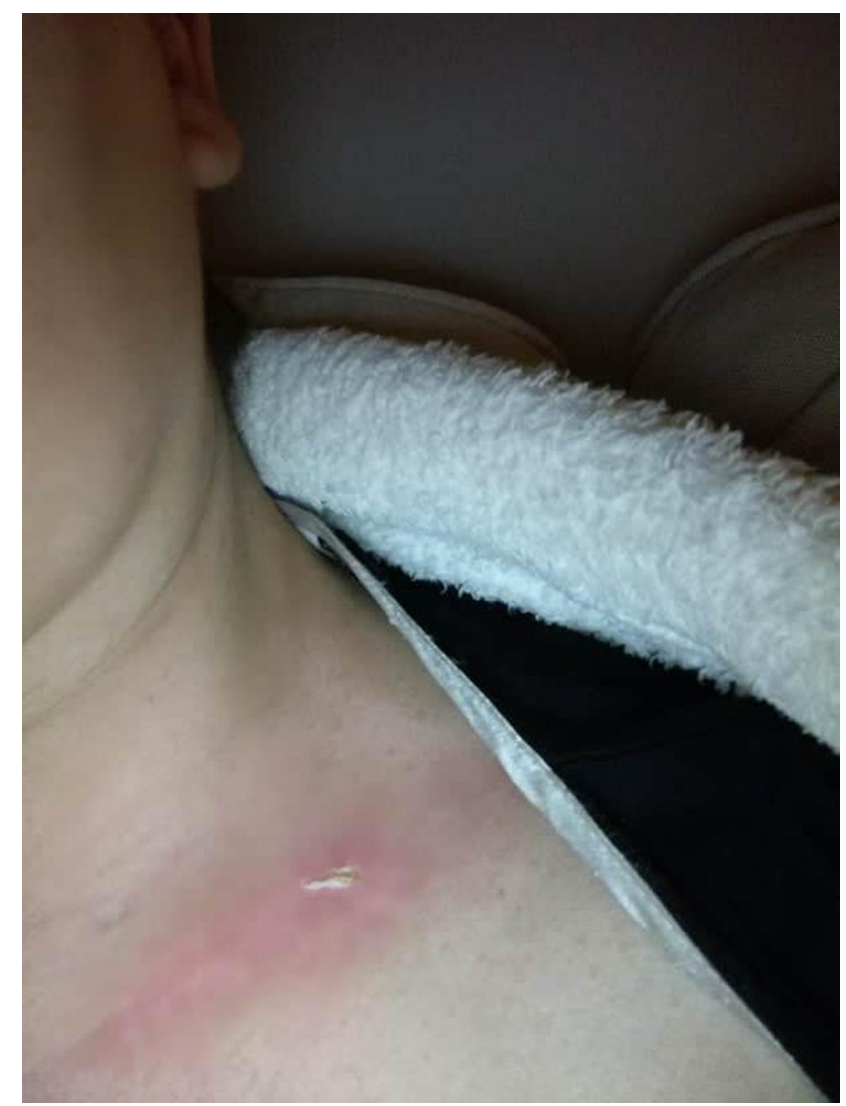

Figure 7 Day 16 post $25^{\text {th }}$ RT. Day 19 Post AQ therapy. Pain score $=0$. ARD gradel.

at six months showed only a minimal scar that was barely visible.

\section{Discussion}

Acute radiodermatitis is a common and almost inevitable consequence of the use of ionizing radiation for breast cancer. Treatment of radiation-induced skin changes can last for four to six weeks, depending on the skin lesion's depth. ${ }^{13}$

Destruction of the skin surface occurs almost immediately after the initial dose of radiation. Every subsequent treatment session results in additional inflammatory cell recruitment, leading to further damage of the living cell layer of the epidermis and disharmony between the dividing cells in the basal layer and epidermal desquamation. There seems to be a direct relationship between the dose of radiation used and the severity of the skin reaction. ${ }^{14}$

Modulation of the inflammatory response is of paramount importance in the management of radiodermatitis. Successful wound healing proceeds through an orderly progression of inflammation, proliferation, and matrix remodeling, with each stage, depending on several 


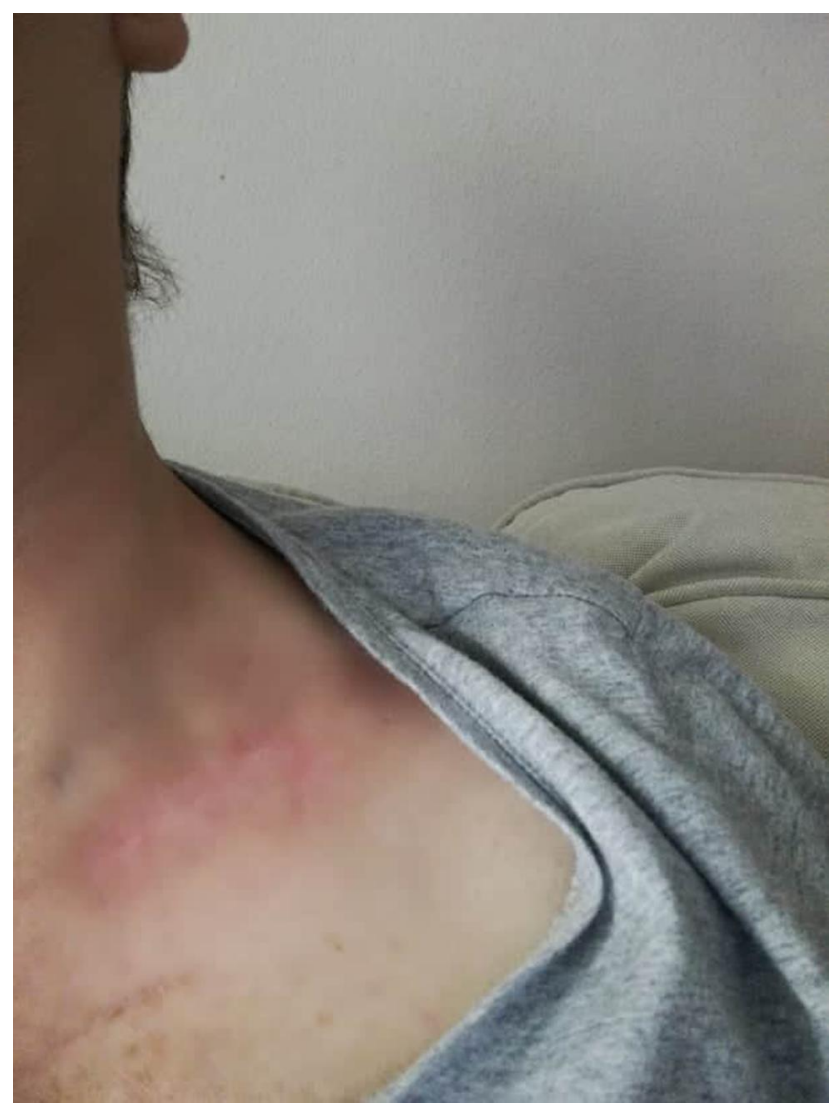

Figure 8 Day 18 post $25^{\text {th }}$ RT. Day 21 Post AQ Therapy. Pain score $=0$. ARD gradel.

interleukins, cytokines, and growth factors. Hence, it stands to reason that the enhancement of these regenerative protein titers by a topical application may optimize the wound-healing response. Although steroids are commonly advocated to treat radiodermatitis, there is a paucity in the literature on the treatment of the condition with these regenerative proteins. Platelet gels, rich in autologous growth factors, have effectively treated chronic cutaneous radiodermatitis, ${ }^{15}$ however, administration of growth factors as creams and solutions has not consistently shown efficacy in their healing properties. ${ }^{16,17}$

The case of the 49-year-old female nurse, is noteworthy in that her condition of grade 3 radiodermatitis resolved very effectively within 21 days, without pain or discomfort. The scar was barely perceptible at six months.

\section{Conclusion}

Skin reactions to treatment with ionizing radiation for breast cancer can be both physically and psychologically

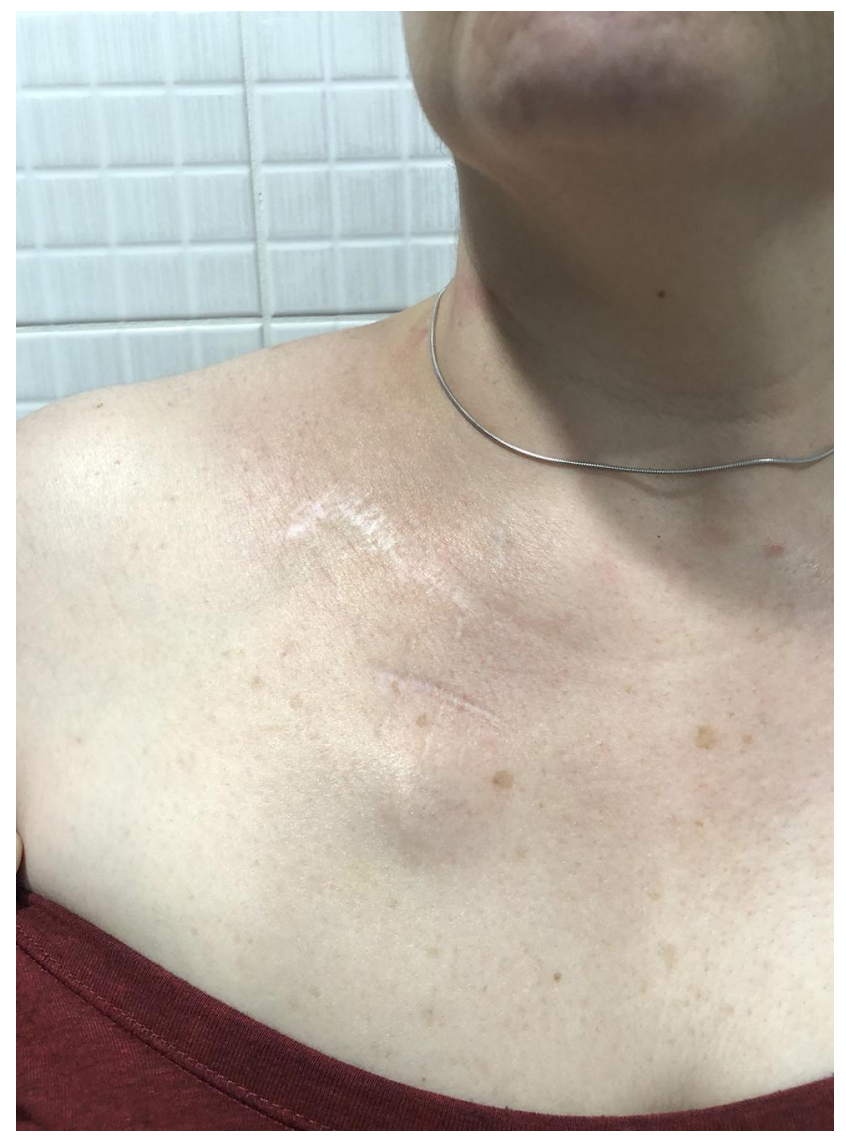

Figure 9 Six months post RT and AQ treatment.

distressing. Hence, swift resolution of radiodermatitis by modulating an orderly wound-healing response may improve patient comfort and minimize scarring and prevent the possible progression to chronic skin lesions. Treatment of a 49-year-old patient with grade 3 radiodermatitis, with a topical preparation of human fibroblast-derived antiinflammatory cytokines, interleukins, and growth factor monotherapy, showed rapid and orderly healing with a significant reduction in pain and virtually no scarring.

This observation warrants further study to uncover the value of such topical preparations of regenerative proteins in managing acute radiodermatitis. In particular, a randomized controlled trial with the AQ human fibroblast-derived preparation is necessary to validate the wound-healing effects in acute radiodermatitis.

\section{Ethics and Consent}

The patient provided the authors with written consent to publish details of her case and all accompanying images. No institutional approval was required for the publications of this case. 


\section{Disclosure}

The authors report no conflicts of interest in this work.

\section{References}

1. Maddock-Jennings W, Wilkinson JM, Shillington D. Novel approaches to radiation-induced skin reactions: a literature review. Complement Ther Clin Pract. 2005;11:224-231. doi:10.1016/j.ctcp.20 05.02.001

2. Chan RJ, Webster J, Chung B, et al. Prevention and treatment of acute radiation-induced skin reactions: a systematic review and meta-analysis of randomized controlled trials. BMC Cancer. 2014; 14:53. doi:10.1186/1471-2407-14-53

3. Deng G, Cassileth BR. Skin Injury: Acute Dermatitis and Chronic Skin Changes. Supportive Care and Quality of Life. 5th ed. Philadelphia, PA: Lippincott Williams \& Wilkins; 2008.

4. Fowble B, Park C, Yuen F. Breast cancer. In: Fowble B, Yom SS, Yuen F, Arron S, editors. Skin Care in Radiation Oncology a Practical Guide. Springer International Publishing; 2016:93-122.

5. National Cancer Institute. Common terminology criteria for adverse events v5.0 NIH; 2018. Available from: https://ctep.cancer.gov/proto coldevelopment/electronic_applications.

6. AFSOS. Toxicité cutanee radio-induit (AFSOS radio-induced cutaneous toxicity). 2014. Available from http://www.afsos.org/fichereferentiel/toxicite-cutanee-rado-induite/.

7. Wong RK, Bendadoun RJ, Boers-Doets CB, et al. Clinical practice guidelines for the prevention and treatment of acute and late radiation reactions from the MASCC Skin Toxicity Study Group. Support Care Cancer. 2013;21(10):2933-2948. doi:10.1007/s00520-013-1896-2

8. Bensadoun RJ, Humbert P, Krutmann J, et al. Daily baseline skin care in the prevention, treatment, and supportive care of skin toxicity in oncology patients: recommendations from a multinational expert panel. Cancer Manag Res. 2013;5:401-408. doi:10.2147/CMAR. S52256
9. Lavery BA. Skin care during radiotherapy: a survey of UK practice. Clin Oncol (R Coll Radiol). 1995;7:184-187. doi:10.1016/S09366555(05)80513-8

10. Hymes SR, Strom EA, Fife C. Radiation dermatitis: clinical presentation, pathophysiology, and treatment 2006. J Am Acad Dermatol. 2006;54:28-46. doi:10.1016/j.jaad.2005.08.054

11. Cooper DM, Yu EZ, Hennessey P, Ko F, Robson MC. Determination of endogenous cytokines in chronic wounds. Ann Surg. 1994;219:688-692. doi:10.1097/00000658-199406000-00012

12. Pierce GF, Tarpley JE, Tseng J, et al. Detection of platelet-derived growth factor (PDGF)-AA in actively healing human wounds treated with recombinant PDGF-BB and absence of PDGF in chronic nonhealing wounds. J Clin Investig. 1995;96:1336. doi:10.1172/JCI1 18169

13. McQuestion M. Evidence based skin care management in radiation therapy. Semin Oncol Nurs. 2006;22:163-173. doi:10.1016/j.soncn. 2006.04.004

14. Salvo N, Barnes E, van Draanen J, et al. Prophylaxis and management of acute radiation-induced skin reactions: a systematic review of the literature. Curr Oncol. 2010;17(4):94-112.

15. Diodato VI, DiMacchia CA, Di Meo T, et al. Platelet gel in cutaneous radiation dermatitis. Clin Trial Support Care Cancer. 2013;21: 287-293. doi:10.1007/s00520-012-1635-0

16. Robson MC, Steed DL, Franz MG. Wound healing: biologic features and approaches to maximize healing trajectories. Curr Probl Surg. 2001;38:72-140. doi:10.1067/msg.2001.111167

17. Park JW, Hwang SR, Yoon IS. Advanced growth factor delivery systems in wound management and skin regeneration. Molecules. 2017;22(8):1259-1279. doi:10.3390/molecules22081259
International Medical Case Reports Journal

\section{Publish your work in this journal}

The International Medical Case Reports Journal is an international, peer-reviewed open-access journal publishing original case reports from all medical specialties. Previously unpublished medical posters are also accepted relating to any area of clinical or preclinical science. Submissions should not normally exceed 2,000 words or 4 published pages including figures, diagrams and references. The manuscript management system is completely online and includes a very quick and fair peer-review system, which is all easy to use. Visit http://www.dovepress.com/testimonials.php to read real quotes from published authors 